Revista de Matemática: TeORía y AplicaCiones 2018 25(1) : 61-78

CIMPA - UCR ISSN: 1409-2433 (PRINT), 2215-3373 (ONLINE)

\title{
SENTIMIENTO BURSÁTIL COMO SISTEMA DINÁMICO
}

\section{THE STOCK MARKET SENTIMENT AS A DYNAMICAL SYSTEM}

\author{
FERnÁn Ulate Montero*
}

Received: 13/Mar/2017; Revised: 27/Sep/2017;

Accepted: $25 /$ Oct/2017

Revista de Matemática: Teoría y Aplicaciones is licensed under a Creative Commons Reconocimiento-NoComercial-Compartirigual 4.0 International License.

Creado a partir de la obra en http://www.revistas.ucr.ac.cr/index.php/matematica

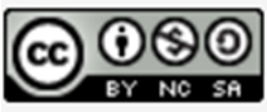

*Escuela de Economía, Universidad de Costa Rica, San José, Costa Rica. E-Mail: ulate80@yahoo.com 


\title{
Resumen
}

Estudiamos un vector de tres entradas, que llamaremos "espíritu bursátil", que refleja el sentimiento sobre si un índice de una Bolsa de Valores va a subir, bajar o permanecer igual. Siguiendo el trabajo sobre la "mentalidad", expuesto en Ulate(2006), dicho sentimiento será visto como un ser vivo, que es una manifestación de una Cultura o Mentalidad en un momento dado. Veremos el cambio de este sentimiento de la misma forma que se ve en animales o plantas. Utilizamos sistemas de ecuaciones diferenciales para describir el cambio y réplica en el tiempo de dicho espíritu, siguiendo la Teoría de Juegos Evolutivos. El sistema de tres ecuaciones será comparado con el sistema de dos ecuaciones, conocido como Lotka Volterra.

Palabras clave: juegos evolutivos; cambio cultural; historia matemática; finanzas; sentimiento bursátil; sistema dinámico.

\begin{abstract}
We study a three entries vector, called "stock market sentiment", that summarizes bear, bull and neutral feeling about the future of the market. Following the paper about "mind or spirit" in Ulate (2006), we consider such sentiment a living being and we will study its change and dynamics using evolutionary game theory. We use a dynamical system of three differential equations to study the change in the vector, and later it will be compared with the Lotka Volterra system with two equations.
\end{abstract}

Keywords: evolutionary games; cultural change; mathematical history; finance; stock market sentiment; dynamical systems.

Mathematics Subject Classification: 91G80.

Concebimos el espíritu, esencialmente como conciencia de sí mismo.

Hegel. 


\section{Introducción}

Dada la importancia que tiene el mercado bursátil en una economía, queremos estudiar, sobretodo, la manera como los sentimientos de los individuos acerca de este mercado interactúan, evolucionan y convergen, utilizando herramientas de teoría de juegos evolutivos y sistemas dinámicos. De hecho, el índice bursátil $\mathrm{S} \& \mathrm{P}$ es un indicador líder, lo que significa que, en general, anticiparía lo que va a pasar en el ciclo económico en U.S.A. El Banco Suizo U.S.B. (2016) [17], demuestra que la variable"Indice Standard \& Poors" (S \& P), sirve para explicar algunas políticas de la Banca Central de Estados Unidos (FED).

En la teoría económica tradicional, se utiliza a menudo el llamado "supuesto de racionalidad", que parte del principio de que los individuos ven, perciben o analizan la realidad tal como es a la hora de tomar sus decisiones. Este supuesto nos lleva fácilmente a modelos que implican equilibrios que no se dan en la vida real. Los individuos analizan y ven la realidad de diferentes maneras, que llamaremos estrategias. Esta diferencia en estrategias es esencial para entender los ciclos y la dinámica política, social y económica a lo largo de la historia. Esto es especialmente cierto en el mundo bursátil.

La relación de los sentimientos acerca de la realidad con la realidad misma tiene una dinámica, que sobre todo en ciencias sociales, posee una gran gama de estudios, pero hasta ahora el uso de la matemática ha sido muy limitado. Es en la concepción hegeliana de la historia, en donde se encuentran una serie de explicaciones dinámicas acerca de la evolución o interacción del espíritu humano, que lejos de ser mecanicistas, realmente toman en cuenta que el espíritu o mentalidad humana es un ser vivo, con múltiples manifestaciones e interacciones con la realidad. En este sentido, nos alejamos de la interpretación tesis, antítesis, síntesis, e interpretamos la concepción hegeliana de la historia como un modelo en el cual el espíritu o mentalidad (ver [24] Ulate, 2006), es esencialmente potencia, cuya capacidad de manifestarse depende de una serie de aspectos. Su manifestación va a producir una interacción y trayectoria que es importante conocer y estudiar, así como la convergencia, si es que la hay, de dicha mentalidad en esta interacción.

Veamos un ejemplo en el mundo bursátil, el cual nos brinda gran cantidad de datos y estadísticas de todo tipo, lo que permitiría estudiar este tema ampliamente. Supongamos que la economía está pasando por una situación de poco crecimiento, alto desempleo y precios bajos en un índice bursátil como el S\& $\mathrm{P}$, y entonces los individuos comienzan a introducir nuevos inventos, maneras de organizarse, tecnología, mercadeo, etc., y algunos inversionistas comienzan a considerar que los precios de la Bolsa han bajado suficiente y se inclinan por una actitud alcista. El aumento en el valor de la riqueza, produce un efecto positivo 
sobre el consumo, la inversión, y el uso de invenciones que antes la economía no permitía. Esto produce un círculo positivo que sigue aumentando el valor de la riqueza, y vista la Bolsa como un juego, este estimula que los individuos inviertan una proporción de la riqueza cada vez más alta en ella. Después de un tiempo, mucho inversionista ha invertido la mayor parte de lo que puede o está dispuesto a invertir en la Bolsa, y comienza a utilizar métodos más riesgosos como las "call options" o endeudamiento "compra al margen". Para rematar, todo este círculo positivo ha llevado a que se espere de la realidad más de lo que esta puede dar, razón por la cual muchas empresas asesoras de inversionistas consideran que la proporción alcistas-bajistas, o "Bull-Bear ratio", es un indicador que debe ser visto con mucho cuidado, puesto que si es muy alto, o sea la gente está muy positiva acerca del futuro de la economía, entonces hay un gran chance de que los precios de las acciones caigan, y por esta razón a menudo este índice se ve como un indicador contrario, "contrarian indicator" ( ver por ejemplo: InvestingAnswers (2016) [12], Yardeni (2016) [27], Tradingsim (2016) [22]). De la misma manera, en ciertos casos, la proporción de volumen transado en opciones llamado "put-call ratio", es un indicador contrario, (ver por ejemplo Stock Chart (2016) [19], investopedia (2016) [16]).

Por otro lado, Alloy y Abrahamson (1988) [3], realizan una serie de estudios en donde se demuestra que los individuos depresivos tienden a valorar subjetivamente una realidad objetiva mejor que los no depresivos, quienes tienden a sobreestimar a su favor dicha realidad y sus posibilidades. En general, los individuos positivos, valoran los juegos de azar de manera diferente a los depresivos, y en estado de euforia consideran que tienen mejores posibilidades de ganar que el dueño del casino. Es por esta razón, que para los casinos, es negocio regalar el licor, ya que el consumo de éste al inicio produce, generalmente, un estado de euforia. Cuando la Bolsa ha venido subiendo, y está muy alta, en general, muchos individuos se sienten alcistas y eufóricos para seguir invirtiendo en ella, y esperan de ésta más de lo que puede dar.

Este cambio en los sentimientos, produce una separación ente el precio y el valor de la riqueza en los mercados, haciendo que una gran cantidad de transacciones en la economía se realicen a precios falsos, y muchos de los indicadores macroeconómicos, como el PIB y sus componentes carezcan de sentido.

Relacionado con lo antes expuesto, está un principio conocido en Sociología como el Teorema de Thomas, (ver Thomas, 1923 [21]) que en resumen dice: "Si las personas definen las situaciones como reales, éstas son reales en sus consecuencias". Lo cual tiene sentido, por ejemplo, al inicio de un periodo de crecimiento en precios, tal como al principio de una burbuja bursátil, pero nunca cuando la definición de la situación le pide a la realidad lo que ésta no puede 
dar, y más bien, el llamado "Teorema", funciona al contrario. La definición de una situación es sobre todo potencia de acción inicial, limitada por la capacidad de los individuos de llevarla a cabo y por la realidad económico-social de satisfacerla. Por último, las consecuencias de dicha definición son el producto de una serie de interacciones y en general de un proceso evolutivo, cuya convergencia debe estudiarse con un sistema dinámico, y por esto la matemática es el instrumento por excelencia.

En el presente trabajo empezamos el análisis con un conjunto de individuos que esencialmente apuestan a que los precios de las acciones van a subir, bajar, o quedar igual, en un futuro cercano (aprox. 6 meses); apuestan a lo que creen. Los individuos son de dos tipos i. aquellos que conocen las diferentes estrategias o técnicas para invertir, y asimismo conocen la valoración de las decisiones bursátiles con dichas técnicas; los individuos a lo largo del tiempo cambian los sentimientos en proporción a los resultados esperados de estos. ii. Los individuos que no conocen nada de bolsa, ni estrategias ni valoraciones, pero comparan su sentimiento con los demás, escogen el sentimiento más exitoso, e imitan el comportamiento de los individuos con dicho sentimiento.

Ambos comportamientos descritos en el párrafo anterior, nos llevan al mismo sistema de ecuaciones dinámicas conocido como replicador dinámico, o ecuación de réplica, en teoría de juegos evolutivos. Dicho sistema tiene tres variables y tres ecuaciones diferenciales. En la sección 4 del trabajo, probamos que el replicador dinámico implica las ecuaciones de Lotka-Volterra, que es conocida en Biología y es un sistema que consta de dos variables y dos ecuaciones diferenciales, este modelo nos lleva tres tipos de interacción en las especies: competencia, cooperativo, o depredador-presa.

La sección 3 del trabajo, estudia el sistema conocido como las ecuaciones Lotka-Volterra depredador-presa, se prueba que dichas ecuaciones implican la ecuación de réplica y se estudian cambios en la velocidad de ajuste. Los resultados anteriores ayudarían a estudiar empíricamente el comportamiento de los individuos a través de la ecuación Lotka-Volterra, que es relativamente sencilla y de dos variables con dos ecuaciones.

\section{Modelo}

Supongamos que en un momento dado $t$ a través de algún tipo de encuesta se pregunta a una población que sigue o está interesado en una Bolsa de Valores, qué opina sobre el futuro de la Bolsa en, por ejemplo, seis meses. Así, el índice S \& P 500 (Standard and Poors), sirve de ejemplo, y los encuestados escogen entre: 
1. Si va a bajar, sentimiento bajista, o "bear".

2. Si va a subir, sentimiento alcista, o "bull" en inglés.

3. Si va a permanecer, sentimiento neutro, o "neutro".

Siguiendo el trabajo "Formalización de una Teoría de la Mentalidad" [24], la proporción de individuos que escoge cada opción se resume a un vector s o "espíritu", que de alguna manera refleja o resume una manifestación cultural que creemos que es muy útil para entender los ciclos económicos y la economía en general.

Llamaremos espíritu del sentimiento bursátil, o espíritu bursátil, al vector s tal que:

$$
\mathbf{s}=\left(\begin{array}{l}
s_{1} \\
s_{2} \\
s_{3}
\end{array}\right) \in \mathbb{R}^{3}
$$

donde $s_{i} \geq 0$ para $i=1,2,3$, además $\sum_{i} s_{i}=1$.

Llamamos $S^{3}=\left\{\mathbf{s} \in \mathbb{R}^{3} / s_{i} \geq 0, s_{1}+s_{2}+s_{3}=1\right\}$, donde las entradas $s_{i}$ de s representan la proporción de cada uno de los sentimientos bajista, alcista o neutro, que llamaremos sentimiento $s_{i}$ o sentimiento $i$. Más tarde supondremos que alguna de las entradas de $\mathbf{s}$, por ejemplo $s_{3}$, es estrictamente positiva.

Si bien es cierto que cada individuo puede sentirse bajista, alcista o neutro, cuando expresa dicho sentimiento de alguna forma y en algún grado comparte una serie de características, hábitos, valoración de la realidad, visión de la economía o el mundo, etc., con la subcultura con que se identifica. Así por ejemplo, los "bulls", ven ciertos canales de televisión, siguen ciertas páginas de internet, ciertos clubes de inversión, tienen ciertos ídolos, etc.

Ahora bien, si en la Teoría de Juegos Evolutivos, se empezó con un juego que juegan los seres vivos, con diferentes características, aquí empezaremos con un juego, con un gran tamaño, sofisticación, cantidad de transacciones y sobre todo con un fuerte efecto sobre la economía de un país o mundo entero.

Los "bulls", "bears", "neutros", son subculturas que comparten o se identifican en algún grado con ciertas características que llamaremos estrategias. Por ejemplo, en inversión bursátil hay estrategias como:

i. Valoración, de acuerdo a parámetros financieros.

ii. Momentum, de acuerdo a como han cambiado los precios.

iii. Proporción del mercado, y cómo ha cambiado.

iv. Dividendos y su crecimiento. 
v. Política económica, la F.E.D.

vi. Ciclo económico.

vii. Series de Fibonacci.

viii. Actividad y tamaño de las empresas.

ix. Nivel de endeudamiento, sobre todo para actividades en dificultad.

x. Calendario, mes o día del daño, etc.

Hay muchas estrategias, algunas poco sofisticadas como seguir los consejos de un comentarista o el horóscopo, y otras muy sofisticadas como el uso de la matemática avanzada o supercomputadoras, que hacen muchas transacciones por segundo, con algún tipo de arbitraje.

Supongamos un número grande $\ell$ de estrategias y llamamos $e_{i}$ la identificación o uso relativo que se le da a cada estrategia dentro de un grupo o conjunto de individuos, así tenemos

$$
\mathbf{e}=\left(\begin{array}{c}
e_{1} \\
\vdots \\
e_{\ell}
\end{array}\right) \quad \mathbf{e} \in \mathbb{R}^{\ell}, \quad e_{i} \geq 0, \quad \sum_{i} e_{i}=1
$$

donde e puede ser visto como el vector de proporciones de individuos de un grupo que escogen cada una de las estrategias, por lo tanto, los individuos son diferentes; o como el vector del uso relativo que cada individuo tiene de cada una de las estrategias, siendo los individuos iguales. Llamamos $\mathbf{e}^{k}$ el vector de estrategias con que se identifica los individuos del grupo con sentimiento bajista, alcista o neutro, con $k=1,2,3$.

Llamamos pago al premio o castigo que recibe o se espera que reciba la estrategia $i$ ante una unidad de individuos (puede ser 1\%) que escogen la estrategia $j$, denotado $u_{i j}$ :

$$
u_{i j} \in \mathbb{R}, \quad i=1, \ldots, \ell ; j=1, \ldots, \ell
$$

$u_{i j}$ es elemento de la matriz $\mathbf{U} \in \mathbb{R}^{\ell \times \ell}$.

Llamamos $a_{i j}$ al pago que recibe el sentimiento $s_{i}$ ante el sentimiento $s_{j}$, $a_{i j}$ puede verse como el cambio en el premio o castigo que recibe el sentimiento $i$, ante un cambio en una unidad (puede ser 1\%) de individuos que escogen el sentimiento $j$ :

$$
a_{i j}=\left(\mathbf{e}^{i}\right)^{\prime} \mathbf{U e}^{j} ; a_{i j} \in \mathbb{R},
$$

$a_{i j}$ es el elemento $i j$ de la matriz $\mathbf{A} \in \mathbb{R}^{3 x 3}$. 
Los animales transmiten a sus descendientes sus características o estrategias a través de los genes. Por ejemplo los bovinos, son herbívoros, tienen ojos a los lados, para una visión de 360 grados, tienen comportamiento gregario, generalmente huyen ante el peligro, a veces después de embestir, no distinguen colores, son muy sensibles a los movimientos de otros animales y en la relación con otros animales como los leones en las sabanas, asumen el papel de presa. El símbolo de Wall Street de un toro (bovino), es un homenaje al sentimiento alcista o "bull".

El oso norteamericano, símbolo de los "bears", o sentimiento bajista, es carnívoro, tiene los ojos adelante para localizar bien la presa, garras grandes, es rapidísimo en sus manotazos, hiberna y espera así que la oportunidad de cacería vuelva después del largo sueño, y en relación con otros animales como el salmón ocupa el lugar del depredador.

La naturaleza ha ideado a través del ciclo de vida y muerte una manera de reproducir los genes más exitosos. En el comportamiento humano, los sentimientos, que son seres vivos porque se replican, cambian y evolucionan, también podemos suponer que se reproducen esencialmente por imitación de aquellos que son más exitosos, medido este logro por sus resultados finales, sin necesidad de conocer los detalles numéricos de cada sentimiento y sus estrategias. Este modelo de imitación, brinda las mismas ecuaciones de réplica que daría un modelo en el cual los individuos conocen los detalles y valoración de los diferentes aspectos de cada sentimiento, aumentando o disminuyendo estos de acuerdo al pago positivo o negativo de cada uno. La transmisión cultural es un asunto complicado, y hay estudios y explicaciones distintas con posiciones ideológicas de diferentes tipos, ver ejemplo Feldman (1976) [6].

El pago total al sentimiento $s_{i}$, usando la matriz A (4) es igual a $\Pi_{s_{i}}$ :

$$
\Pi_{s_{i}}=\sum_{j} a_{i j} s_{j}=(\mathbf{A s})_{i},(\text { elemento } i \text { del vector } \mathbf{A s})
$$

i.e., el pago al sentimiento $i$ es la suma del pago $i$ ante cada uno de los sentimientos $a_{i j}$ multiplicado por la proporción de cada uno de esos sentimientos, o si se quiere por la probabilidad de encontrarse con individuos del sentimiento $j$.

El pago promedio para todos los sentimientos es igual a:

$$
W=\sum_{j} \sum_{i} s_{i} a_{i j} s_{j}=\mathbf{s}^{\prime} \mathbf{A} \mathbf{s}
$$


Taylor \& Jocker (1978) [20] introducen la "ecuación de réplica", basados en el trabajo pionero de J. Maynard Smith \& G.R. Price (1973) [15], donde el pago está asociado a la capacidad de tener descendientes. Dicha ecuación, para el caso del espíritu bursátil, nos daría que el crecimiento per cápita de un sentimiento $i$ es igual a la diferencia de pago entre dicha estrategia y el pago promedio:

$$
\frac{\dot{s_{i}}}{s_{i}}=(\mathbf{A s})_{i}-\mathbf{s}^{\prime} \mathbf{A} \mathbf{s}, i=1,2,3, \text { con } \dot{s_{i}}=\frac{d s_{i}}{d t}
$$

conocida como la ecuación de réplica.

En el caso de individuos cuya permanencia o cambio de sentimiento no depende del pago (7), sino de imitación con algún tipo de protocolo, tenemos que si los individuos que quieren cambiar tratan de imitar los sentimientos más exitosos en una proporción a ese éxito, entonces volveremos a encontrar esencialmente dicha ecuación. En efecto, supongamos que tenemos dos individuos que se encuentran aleatoriamente y comparan el pago de sus sentimientos $\left(i^{*}, i\right)$.

Si $(\mathbf{A s})_{i^{*}}>(\mathbf{A s})_{i}$, el individuo con $i^{*}$, para no perder, suponemos que conserva su sentimiento y el de $i$ mejoraría si se pasa a $i^{*}$.

$\mathrm{Si}(\mathbf{A s})_{i^{*}}<(\mathbf{A s})_{i}$, entonces el individuo con $i^{*}$, mejoraría si se pasa a $i, \mathrm{y}$ el de $i$ conserva el suyo, pues perdería si cambia.

Suponiendo que los individuos cambian sentimiento en proporción a la mejora entre los dos pagos, entonces el cambio en el sentimiento $i^{*}$ ante encuentros con individuos del sentimiento $i,\left(\frac{d s_{i^{*}}}{d t}\right)_{i}$, es igual a dicha mejora multiplicada por la probabilidad de que un individuo del sentimiento $i^{*}$ se encuentra con otro del $i\left(s_{i^{*}} \cdot s_{i}\right)$ :

$$
\left(\frac{d s_{i^{*}}}{d t}\right)_{i}=\left[(\mathbf{A} \mathbf{s})_{i^{*}}-(\mathbf{A} \mathbf{s})_{i}\right]\left(s_{i^{*}} \cdot s_{i}\right) .
$$

El cambio total en el sentimiento $i^{*},\left(\frac{d s_{i *}}{d t}\right)$, es la suma del cambio en dicho sentimiento, ante los otros sentimientos, $\frac{d s_{i *}}{d t}=\sum_{i}\left(\frac{d s_{i^{*}}}{d t}\right)_{i}$ sumando (8) para todo $i$ y dividiendo entre $s_{i^{*}}$ :

$$
\left(\frac{\frac{d s_{i^{*}}}{d t}}{s_{i^{*}}}\right)=(\mathbf{A} \mathbf{s})_{i^{*}} \sum_{i} s_{i}-\sum_{i} s_{i}(\mathbf{A} \mathbf{s})_{i}
$$

puesto que $\sum_{i} s_{i}=1, \sum_{i} s_{i}(\mathbf{A} \mathbf{s})_{i}=\mathbf{s}^{\prime} \mathbf{A} \mathbf{s}$, entonces $\frac{\frac{d s_{i}}{d t}}{s_{i^{*}}}=(\mathbf{A} \mathbf{s})_{i^{*}}-\mathbf{s}^{\prime} \mathbf{A} \mathbf{s}$, que generalizado para $i^{*}=1,2,3$ conlleva a la ecuación (7).

La ecuación (7) cumple con el hecho de que $\dot{s}_{1}+\dot{s}_{2}+\dot{s}_{3}=0$, como se puede comprobar fácilmente. 


\section{La ecuación de Lotka-Volterra}

Años antes de que Taylor \& Jonker (1978) [20] introdujeran la ecuación de réplica (7), A.J. Lotka (1956, original de 1924) [13], introdujo un sistema de ecuaciones diferenciales en dos dimensiones, y V. Volterra (1931) [25] utilizó el mismo modelo para explicar la composición de peces observada por pescadores en el Mar Adriático, después de la Primera Guerra Mundial.

Dicho modelo, conocido como el sistema Lotka-Volterra, depredador-presa, se presenta a continuación:

$$
\begin{aligned}
& \dot{y}_{1}=y_{1}\left(\alpha_{1}-\beta_{1} y_{2}\right)=f^{\prime}\left(y_{1}, y_{2}\right) \\
& \dot{y}_{2}=y_{2}\left(-\alpha_{2}+\beta_{2} y_{1}\right)=f^{2}\left(y_{1}, y_{2}\right)
\end{aligned}
$$

con $y_{i}, \alpha_{i}, \beta_{i} \in \mathbb{R} ; y_{i} \geq 0 ; \alpha_{i}, \beta_{i}>0$ para $i=1,2$, donde $y_{1}$ es la cantidad de la especie presa, $y_{2}$ es la cantidad de la especie depredadora, $\alpha_{1}$ es el crecimiento proporcional de la especie presa en ausencia de depredadores, $\alpha_{2}$ es el crecimiento proporcional de la especie depredadora (mortalidad) en ausencia de la especie presa (que sirve de comida), $\beta_{1}$ es la manera como una unidad depredadora disminuye el crecimiento proporcional de presas, $\beta_{2}$ es la manera como una unidad presa aumenta el crecimiento proporcional de depredadores.

El equilibrio de (9) se da en $\dot{y}_{i}=0, i=1,2$, así tenemos dos equilibrios:

$$
\begin{gathered}
y_{1}^{e}=y_{2}^{e}=0 \\
\alpha_{1}-\beta_{1} y_{2}^{e}=0 \Rightarrow y_{2}^{e}=\frac{\alpha_{1}}{\beta_{1}} \\
\alpha_{2}-\beta_{2} y_{1}^{e}=0 \Rightarrow y_{1}^{e}=\frac{\alpha_{2}}{\beta_{2}} .
\end{gathered}
$$

Para el caso en que $y_{1}>0$, y $y_{2}>0$, tenemos

$$
\frac{\dot{y}_{1}}{y_{1}} \frac{\dot{y}_{2}}{y_{2}}-\frac{\dot{y}_{2}}{y_{2}} \frac{\dot{y}_{1}}{y_{1}}=0 \text {. }
$$

Reacomodando y utilizando 9, la expresión anterior nos lleva a:

$$
\frac{\alpha_{2}-\beta_{2} y_{1}}{y_{1}} \dot{y}_{1}+\frac{\alpha_{1}-\beta_{1} y_{2}}{y_{2}} \dot{y}_{2}=0
$$

de donde

$$
\alpha_{2} \frac{\dot{y}_{1}}{y_{1}}-\beta_{2} \dot{y}_{1}+\alpha_{1} \frac{\dot{y}_{2}}{y_{2}}-\beta_{1} \dot{y}_{2}=0
$$

o sea

$$
\frac{d\left(\alpha_{2} \ln y_{1}-\beta_{2} y_{1}+\alpha_{1} \ln y_{2}-\beta_{1} y_{2}\right)}{d t}=0
$$


En Acuña \& Ulate (2008) [2], pag. 178, se define una función $V$ que nos ayuda a describir el comportamiento de (9): $V:$ int $\mathbb{R}_{+}^{2} \rightarrow \mathbb{R}$ con int $\mathbb{R}_{+}^{2}=\left\{\left(y_{1}, y_{2}\right) \in \mathbb{R}^{2}, y_{i}>0, i=1,2\right\}$, tal que

$$
V\left(y_{1}, y_{2}\right)=\beta_{2}\left(y_{1}^{e} \ln y_{1}-y_{1}\right)+\beta_{1}\left(y_{2}^{e} \ln y_{2}-y_{2}\right) .
$$

Utilizando (11), obtenemos que $V$ es la expresión entre paréntesis en (12), con lo que (12) se puede expresar $\frac{d V}{d t}=0$ y se obtiene:

$$
V\left(y_{1}(t), y_{2}(t)\right)=k \text {. }
$$

El valor de la constante $k$ de $V$ determina la posición de órbitas cerradas, alrededor del punto de equilibrio $y^{e}=\left(y_{1}^{e}, y_{2}^{e}\right)$.

Utilizando el Teorema de Taylor, podríamos encontrar una expansión lineal en el vecindario de $y^{e}=\left(y_{1}^{e}, y_{2}^{e}\right)$ en (11). En equilibrio

$$
\begin{aligned}
& \dot{y}_{1}=f^{1}=0 \\
& \dot{y}_{2}=f^{2}=0
\end{aligned}
$$

y la nueva función $\left(f_{1}, f_{2}\right)$ linealizada sería

$$
\dot{z}=J^{e} \cdot z,
$$

donde $J^{e}=\left|\frac{\partial\left(f^{1}, f^{2}\right)}{\partial y_{1} \partial y_{2}}\right|_{y^{e}} \in \mathbb{R}^{2 \times 2}$ es la matriz jacobiana de $\left(f^{1}, f^{2}\right)$ valorado en $y^{e}, z=\left(y-y_{e}\right)$ donde $y=\left(\begin{array}{l}y_{1} \\ y_{2}\end{array}\right)$ y claramente $d y=d z$, con lo que estudiar (15) nos ayuda a entender el comportamiento de (9), sobre todo en estabilidad y tipo de equilibrio.

Ahora,

$$
J=\left|\begin{array}{ll}
\frac{\partial f^{1}}{\partial y_{1}} & \frac{\partial f^{1}}{\partial y_{2}} \\
\frac{\partial f^{2}}{\partial y_{1}} & \frac{\partial f^{2}}{\partial y_{2}}
\end{array}\right|=\left|\begin{array}{cc}
\left(\alpha_{1}-\beta_{1} y_{2}\right) & -\beta_{1} y_{1} \\
\beta_{2} y_{2} & \left(-\alpha_{2}+\beta_{2}\right) y_{1}
\end{array}\right|
$$

que valorado en $y_{1}^{e}=\alpha_{2} / \beta_{2}, y_{2}^{e}=\alpha_{1} / \beta_{1}$ se obtiene

$$
J^{e}=\left|\begin{array}{cc}
0 & -\beta_{1} \frac{\alpha_{2}}{\beta_{2}} \\
\beta_{2} \frac{\alpha_{1}}{\beta_{1}} & 0
\end{array}\right| .
$$

Ls solución general para (15), cuando se tienen raíces características para $J^{e}$, reales y distintas $\left(\lambda_{1}, \lambda_{2}\right)$, es

$$
z(t)=\mathbf{k}_{1} c_{1} e^{\lambda_{1} t}+\mathbf{k}_{2} c_{2} e^{\lambda_{2} t}
$$


donde

$$
z(t)=\left(\begin{array}{l}
z_{1}(t) \\
z_{2}(t)
\end{array}\right)
$$

con $\mathbf{k}_{1}, \mathbf{k}_{2}$ vectores característicos asociados a $\lambda_{1}, \lambda_{2}$ respectivamente.

Si las raíces son complejas, i.e $\lambda_{1}, \lambda_{2}=a \pm b_{i}$ se tiene

$$
z(t)=\mathbf{k}_{1} c_{3} e^{a t} \cos \left(b t+c_{4}\right)+\mathbf{k}_{2} c_{3} e^{a t} \sin \left(b t+c_{4}\right)
$$

donde $\mathbf{k}_{1}$ y $\mathbf{k}_{2}$ son vectores característicos asociados a la parte real y a la parte imaginaria, respectivamente, del vector característico asociado a la raíz característica $\lambda_{1}, \mathrm{y} c_{3}, c_{4}$ son constantes reales con $c_{4} \in[0,2 \pi]$.

Las raíces características de $J^{e}$ son $\lambda_{i}= \pm i\left(\alpha_{1} \cdot \alpha_{2}\right)^{\frac{1}{2}}$ con lo que se obtiene para (15) la solución

$$
z(t)=\mathbf{k}_{1} c_{3} \cos \left(\sqrt{\alpha_{1} \alpha_{2}} t+c_{4}\right)+\mathbf{k}_{2} c_{3} \sin \left(\sqrt{\alpha_{1} \alpha_{2}} t+c_{4}\right) .
$$

Esta linealización nos lleva a que $y_{e}$ está rodeado de órbitas cerradas con período igual a $\frac{2 \pi}{\sqrt{\alpha_{1} \alpha_{2}}}$.

Partiendo de (9), definamos un nuevo sistema con $s_{3}=\frac{1}{y_{1}+y_{2}+1}>0$,

i. $\dot{y}_{1}=f^{1}\left(y_{1}, y_{2}\right) \cdot s_{3}=y_{1}\left(\alpha_{1}-\beta_{1} y_{2}\right) \cdot s_{3}=g^{1}\left(y_{1}, y_{2}\right)$

ii. $\dot{y}_{2}=f^{2}\left(y_{1}, y_{2}\right) \cdot s_{3}=y_{2}\left(-\alpha_{2}+\beta_{2} y_{1}\right) \cdot s_{3}=g^{2}\left(y_{1}, y_{2}\right)$.

La nueva matriz jacobiana es

$$
J=\left[\begin{array}{ll}
\frac{\partial g^{1}}{\partial y_{1}} & \frac{\partial g^{1}}{\partial y_{2}} \\
\frac{\partial g^{2}}{\partial y_{1}} & \frac{\partial g^{2}}{\partial y_{2}}
\end{array}\right]
$$

En equilibrio

$$
\begin{aligned}
& f^{1}=y_{1}\left(\alpha_{1}-\beta_{1} y_{2}\right)=0 \\
& f^{2}=y_{2}\left(-\alpha_{2}+\beta_{2} y_{1}\right)=0 .
\end{aligned}
$$

Utilizando $\frac{\partial s_{3}}{\partial y_{1}}=\frac{\partial s_{3}}{\partial y_{2}}=-s_{3}^{2}$, entonces la nueva matriz jacobiana valorada en equilibrio es

$$
J_{n}^{e}=\left[\begin{array}{cc}
0 & -\beta_{1} \frac{\alpha_{2}}{\beta_{2}} \cdot s_{3}^{e} \\
\beta_{2} \frac{\alpha_{1}}{\beta_{1}} \cdot s_{3}^{e} & 0
\end{array}\right],
$$

donde

$$
s_{3}^{e}=\frac{1}{\left(\alpha_{2} / \beta_{2}\right)+\left(\alpha_{1} / \beta_{1}\right)+1}
$$


lleva a la ecuación característica

$$
\lambda^{2}+\alpha_{1} \alpha_{2}\left(s_{3}^{e}\right)^{2}=0
$$

Las raíces características son $\lambda_{i}= \pm i \sqrt{\alpha_{1} \alpha_{2}} s_{3}^{e}$.

Tenemos las mismas órbitas cerradas de (15), pero ahora el período es

$$
\frac{2 \pi}{\sqrt{\alpha_{1} \alpha_{2}}} \cdot\left(\frac{\alpha_{2}}{\beta_{2}}+\frac{\alpha_{1}}{\beta_{1}}+1\right) .
$$

Del resultado anterior para $J_{n}^{e}$, se obtiene $J_{n}^{e}=s_{3}^{e} J^{e}$. Sea $H(\delta), \delta \in[0,1]$ una matriz tal que $H(\delta)=\left(1+\left(s_{3}^{e}-1\right) \cdot \delta\right) J^{e}, H(0)=J^{e}, H(1)=J_{n}^{e}$.

Vistas $f=\left(f^{1}, f^{2}\right)$ y $g=\left(g^{1}, g^{2}\right)$ linealizadas como campos vectoriales definidos sobre una curva cerrada, entonces son homotópicos (como relación de equivalencia, ver Farkas (1994) [5]).

Sea la transformación $T: \mathbb{R}_{+}^{2} \rightarrow S^{3}, T\left(y_{1}, y_{2}\right)=\left(s_{1}, s_{2}, s_{3}\right)$, tal que

$$
\begin{aligned}
s_{1} & =\frac{y_{1}}{y_{1}+y_{2}+1} \\
s_{2} & =\frac{y_{2}}{y_{1}+y_{2}+1} \\
s_{3} & =\frac{1}{y_{1}+y_{2}+1} .
\end{aligned}
$$

Puesto que $s_{1}=y_{1} \cdot s_{3}, s_{2}=y_{2} \cdot s_{3}$ y que

$$
\begin{aligned}
& \dot{s}_{3}=\frac{-\left(\dot{y}_{1}+\dot{y}_{2}\right)}{\left(y_{1}+y_{2}+1\right)^{2}}=-s_{3}^{2}\left(\dot{y}_{1}+\dot{y}_{2}\right) \\
& \dot{s}_{1}=s_{3} \dot{y}_{1}+y_{1} \dot{s}_{3} \\
& \dot{s}_{2}=s_{3} \dot{y}_{2}+y_{2} \dot{s}_{3}
\end{aligned}
$$

entonces, utilizando el módelo de Lotka-Volterra, depredador presa con ajuste $s_{3}$ (16), obtenemos:

$$
\begin{aligned}
& \dot{s}_{1}=s_{1}\left[\left(\alpha_{1} s_{3}-\beta_{1} s_{2}\right)-\left(\alpha_{1} s_{1} s_{3}-\beta_{1} s_{1} s_{2}-\alpha_{2} s_{2} s_{3}+\beta_{2} s_{2} s_{1}\right)\right] \\
& \dot{s}_{2}=s_{2}\left[\left(-\alpha_{2} s_{3}+\beta_{2} s_{1}\right)-\left(\alpha_{1} s_{1} s_{3}-\beta_{1} s_{1} s_{2}-\alpha_{2} s_{2} s_{3}+\beta_{2} s_{2} s_{1}\right)\right] \\
& \dot{s}_{3}=s_{3}\left[-\left(\alpha_{1} s_{1} s_{3}-\beta_{1} s_{1} s_{2}-\alpha_{2} s_{2} s_{3}+\beta_{2} s_{2} s_{1}\right)\right]
\end{aligned}
$$

$\mathrm{y}$

$$
\left[\begin{array}{c}
\dot{s}_{1} / s_{1} \\
\dot{s}_{2} / s_{2} \\
\dot{s}_{3} / s_{3}
\end{array}\right]=\left[\begin{array}{ccc}
0 & -\beta_{1} & \alpha_{1} \\
\beta_{2} & 0 & -\alpha_{2} \\
0 & 0 & 0
\end{array}\right]\left[\begin{array}{l}
s_{1} \\
s_{2} \\
s_{3}
\end{array}\right]-\mathbf{s}^{\prime}\left[\begin{array}{ccc}
0 & -\beta_{1} & \alpha_{1} \\
\beta_{2} & 0 & -\alpha_{2} \\
0 & 0 & 0
\end{array}\right] \mathbf{s} .
$$


O sea para

$$
\mathbf{A}=\left[\begin{array}{ccc}
0 & -\beta_{1} & \alpha_{1} \\
\beta_{2} & 0 & -\alpha_{2} \\
0 & 0 & 0
\end{array}\right]
$$

se tiene

$$
\dot{s}_{i}=s_{i}\left[(\mathbf{A s})_{i}-\mathbf{s}^{\prime} \mathbf{A} \mathbf{s}\right], i=1,2,3,
$$

que es la ecuación de la réplica (7) para $\mathbf{A}$ dado.

\section{La Ecuación de Lotka-Volterra (generalizada) y la ecuación de réplica}

Generalizando la ecuación Lotka-Volterra depredador-presa para incluir el efecto que un determinado sentimiento tenga sobre sí mismo, se tiene, para $y=\left(y_{1}, y_{2}\right) \in \mathbb{R}_{+}^{2}$,

$$
\begin{aligned}
& \dot{y}_{1}=\frac{d y_{1}}{d t}=y_{1}\left(d_{13}+d_{11} y_{1}+d_{12} y_{2}\right)=f^{1}\left(y_{1}, y_{2}\right) \\
& \dot{y}_{2}=\frac{d y_{2}}{d t}=y_{2}\left(d_{23}+d_{21} y_{1}+d_{22} y_{2}\right)=f^{2}\left(y_{1}, y_{2}\right)
\end{aligned}
$$

donde $a_{11}, a_{22}$ es el efecto que un sentimiento específico tiene sobre sí mismo; se tienen tres casos:

i. $d_{12}$ y $d_{21}$ positivos, el sistema es cooperativo.

ii. $d_{12}$ y $d_{21}$ negativos, el sistema es competitivo.

iii. $d_{12} d_{21}<0$, el sistema es depredador-presa.

Partiendo de la ecuación de réplica (7) y suponiendo $s_{3}>0$,

$$
\dot{s}_{i}=s_{i}\left[(\mathbf{A s})_{i}-\mathbf{s}^{\prime} \mathbf{A} \mathbf{s}\right], i=1,2,3 .
$$

Llamamos $y_{1}=s_{1} / s_{3}, y_{2}=s_{2} / s_{3}, y_{3}=1$, entonces, para $i=1,2,3$ :

$$
\begin{aligned}
\dot{y}_{i} & =\frac{s_{3} \dot{s}_{i}-s_{i} \dot{s}_{3}}{s_{3}^{2}} \\
& =\frac{s_{3} s_{i}\left[(\mathbf{A} \mathbf{s})_{i}-\mathbf{s}^{\prime} \mathbf{A} \mathbf{s}\right]-s_{i} s_{3}\left[(\mathbf{A} \mathbf{s})_{3}-\mathbf{s}^{\prime} \mathbf{A} \mathbf{s}\right]}{s_{3}^{2}} \\
& =\frac{s_{i}}{s_{3}}\left[(\mathbf{A} \mathbf{s})_{i}-(\mathbf{A} \mathbf{s})_{3}\right] \\
& =y_{i}\left[\left(a_{i 1} s_{1}+a_{i 2} s_{2}+a_{i 3} s_{3}\right)-\left(a_{31} s_{1}+a_{32} s_{2}+a_{33} s_{3}\right)\right] \\
& =y_{i}\left[\left(a_{i 1}-a_{31}\right) \frac{s_{1}}{s_{3}}+\left(a_{i 2}-a_{32}\right) \frac{s_{2}}{s_{3}}+\left(a_{i 3}-a_{33}\right) \frac{s_{3}}{s_{3}}\right] s_{i} .
\end{aligned}
$$


Llámese $\left(a_{i j}-a_{3 j}\right)=d_{i j}$, entonces se tiene:

$$
\begin{aligned}
& \dot{y}_{1}=y_{1}\left(d_{13}+d_{11} y_{1}+d_{12} y_{2}\right) \cdot s_{3}=g^{1}\left(y_{1}, y_{2}\right) \\
& \dot{y}_{2}=y_{1}\left(d_{23}+d_{21} y_{1}+d_{22} y_{2}\right) \cdot s_{3}=g^{2}\left(y_{1}, y_{2}\right)
\end{aligned}
$$

que es la ecuación (17) con un cambio en la velocidad de ajustes $\left(s_{3}\right)$.

En la sección anterior vimos que para el sistema Lotka-Volterra depredadorpresa, como las órbitas eran cerradas, a lo largo de dicha curva cerrada los campos vectoriales linealizados $f=\left(f_{1}, f_{2}\right)$, y $g=\left(g_{1}, g_{2}\right)$ son homotópicos, $g=s_{3} \cdot f$. También podemos encontrar una serie de propiedades relacionadas con la equivalencia entre los modelos (17) y 19). Dicha equivalencia fue estudiada originalmente por Hofbauer(1981) [9] para $n$ variables (replicador) y $(n-1)$ variables (Lotka-Volterra). En un trabajo posterior Bomze (1983) [4], aprovecha dicha equivalencia para clasificar los flujos de dichas ecuaciones o sistemas.

\section{Conclusiones}

Partir del supuesto de que los individuos ven la realidad de diferentes formas, que llamamos estrategias, nos lleva a comportamientos dinámicos, a veces cíclicos, que permiten comprender mejor el ciclo económico.

Dada la importancia que tienen las bolsas de valores, como indicadores líderes en el ciclo económico y en la política de la Banca Central de la Reserva Federal, estudiar estadísticamente los modelos (17) y 19), podría ayudarnos a entender mejor la economía.

La ecuación de Lotka-Volterra, reduce el sistema de 3 ecuaciones en tres dimensiones a un sistema de 2 ecuaciones en dos dimensiones, y facilita el estudio empírico-estadístico y el conocimiento sobre las órbitas que ahora se dan en el plano.

El ciclo económico es un asunto complejo que se ha visto de muchas maneras. A finales del siglo XX y principios del siglo XXI, la política fiscal y monetaria ha sido usada para suavizar o disminuir las fluctuaciones en dicho ciclo. Esta política debe ser analizada en un sentido amplio, puesto que si bien es cierto estas fluctuaciones producen recesiones, desempleo, y en general, sufrimiento, este sufrimiento debe ser compensado sin afectar las bondades de la destrucción creativa (Schumpeter 1942) [18], en el largo plazo, sobre la economía en general, y específicamente sobre la tecnología. 


\section{Referencias}

[1] Acuña, O.; Ulate, F. (1992) "Equilibrio de Precios y Óptimo de Pareto en Intercambio", Revista de Matemática: Teoría y Aplicaciones 3(1):

[2] Acuña, O.; Ulate, F. (2008) "Adam Smith y Sistemas Dinámicos", Revista de Ciencias Económicas 26(1): 171-185.

[3] Alloy, L.; Abrahamson, L. (1988) “Depressive Realism”, in: L.B. Alloy (Ed.) Cognitive Processes in Depression, Guilford Press, New York: 441485 .

[4] Bomze, I.M. (1983) "Lotka-Volterra Equations and Replicator Dynamics: A Two Dimensional Classification”, Biological Cybernetics. 48: 201-211.

[5] Farkas, M. (1994) Periodic Motions. Springer Verlag, New York.

[6] Feldman, M.; Cavalli-Sforza, L. (1976) "Cultural and Biological Evolutionary Processes, Selection for a Trait Under Complex Transmission”, Theoretical Population Biology 9:238-259

[7] Hegel, J. (1928) "Filosofía de la historia universal", Rev. Occidente. Madrid.

[8] Hegel, J. (1966) Fenomenología del Espíritu. Fondo de Cultura Económica, México D.F.

[9] Hofbauer, J. (1981) "On the Ocurrence of Limit Cycles in the VolterraLotka Equation”, Nonlinear Analysis, Methods \& Applications 5(9): 10031007.

[10] Hofbauer, J.; Schuster, P.; Sigmund, K. (1979) "A Note On Evolutionary Stable Strategies and Game Dynamics", Journal Theoretical Biology 81: 609-612.

[11] Hofbauer, J.; Sigmund, K. (1998) Evolutionary Games and Population Dynamics. Cambridge University Press, Cambridge.

[12] InvestingAnswers (2016) "Bull/Bear Ratio", en: http://www. investinganswers.com/financial-dictionary/ stock-market/bullbear-ratio-1775.

[13] Lotka, A. (1956) Elements of Mathematical Biology. Dover Publications, New York. 
[14] Maynard, J. (1982) Evolution and the Theory of Games. Cambridge University Press, Cambridge.

[15] Maynard, J.; Price, G. (1973) “The Logic of Animal Conflict”, Nature 246: $15-18$.

[16] Murphy, C. (2016) "What is the put-call ratio and why should I pay attention to it?", en: https://www.investopedia.com/ask/ answers/06/putcallratio.asp

[17] Ro, S. (2016) "UBS: The stock market guides the FED", Yahoo Finance, May 9, 2016. Tomado de finance.yahoo.com/news/ ubs-equities-stocks-guide-fed-dots-monetary -policy-201427082.html

[18] Schumpeter, J. (1996) Capitalismo, Socialismo y Democracia. Folio, Barcelona.

[19] Stock Charts (2016) http://stockcharts.com/school/ doku.php?id=chart--school:technical--indicators: put--call--ratio

[20] Taylor, P.; Jonker, L. (1978) "Evolutionary Stable Strategies and Game Dynamics", Mathematical Biosciences 40(1 y 2): 145-156.

[21] Thomas, W. (1923) The Unadjusted Girl: With Cases and Standpoint for Behavior Analysis. Little, Brown and Company, Boston.

[22] TradingSim (s.f) "Bull Bear Ratio Definition and Formula", en: https://tradingsim.com/blog/bull-bear-ratio/.

[23] Ulate, F. (1988) "El Modelo Neoclásico y una Política de Crecimiento para Costa Rica”, Revista de Ciencias Económicas 8(1): 39-53.

[24] Ulate, F. (2006) "Formalización de una Teoría de la Mentalidad", Revista de Matemática: Teoría y Aplicaciones 13(1): 53-80

[25] Volterra, V. (1931) Leçons sur la Théorie Mathématique de la Lutte pour la Vie. Gauthier-Villars, Paris.

[26] Weibull, J. (1995) Evolutionary Game Theory. MIT Press, Cambridge.

[27] Yardeni, E.; Abbott, J.; Johnson, D.; Quintana, M. (2017) "Stock Market Indicators: Bull/Bear Ratios", en: http: / /www • yardeni . com/pub/ stmktbullbear.pdf. 
[28] Zeeman, E. (1981) "Dynamics of the Evolution of Animal Conflicts", Journal of Theoretical Biology 89(2): 249-270.

Rev.Mate.Teor.Aplic. (ISSN print: 1409-2433; online: 2215-3373) Vol. 25(1): 61-78, Jan-Jun 2018 